\title{
Pursuit differential games with state constraints
}

\author{
Cardaliaguet Pierre*, Quincampoix Marc ${ }^{\dagger} \&$ Saint-Pierre Patrick ${ }^{\ddagger}$
}

January 20, 2004

\begin{abstract}
We prove the existence of a value for pursuit games with state-constraints. We also prove that this value is lower semi-continuous.

\section{Résumé}

Nous démontrons l'existence d'une valeur pour les jeux de poursuite, avec des contraintes sur état et prouvons que cette fonction-valeur est semicontinue inférieurement.
\end{abstract}

In this paper, we intend to prove that two-players differential games with state-constraints have a value.

We investigate a differential game where the first player, called Ursula, playing with $u$, controls a first system

$$
y^{\prime}(t)=g(y(t), u(t)), \quad u(t) \in U
$$

and has to ensure the state constraint $y(t) \in K_{U}$ to be fulfilled, while the second player, called Victor, playing with $v$, controls a second system

$$
z^{\prime}(t)=h(z(t), v(t)), \quad v(t) \in V
$$

and has to ensure the state constraint $z(t) \in K_{V}$. The first player aims the state of the full system $(y(\cdot), z(\cdot))$ at avoiding a target as long as possible while the second player aims the state of the system at reaching this target in minimal time.

\footnotetext{
*Centre de recherche Viabilité, Jeux, Contrôle, Université Paris IX Dauphine, Place du Maréchal de Lattre de Tassigny, 75775 Paris Cedex

${ }^{\dagger}$ Département de Mathématiques, Université de Bretagne Occidentale, 6 Avenue Victor Le Gorgeu F-29200 Brest

$\ddagger$ Centre de recherche Viabilité, Jeux, Contrôle, Université Paris IX Dauphine, Place du Maréchal de Lattre de Tassigny, F-75775 Paris Cedex
} 
This game is known as the pursuit game. Most of the examples and results of the early theory for differential games are concerned with this problem (for several examples and for methods of explicit resolution, see Isaacs [25], Flynn [19], Breakwell [9], Bernhard [8]).

As usually in differential game theory, one can define two value functions for the game, the upper one and the lower one. The purpose of this paper is to give some conditions on the system under which the pursuit game has a value, i.e., that the upper value function is equal to the lower value function. We have to face two difficulties: the presence of state-constraints, and the fact that the value functions can be discontinuous. Let us point out that in most examples studied in Isaacs' book [25] one has to face at least one of the difficulties, and often both.

In the early 70 , the question of the existence of a value for pursuit games is the aim of several papers (Varaiya \& Lin [36], Osipov [28], Friedman [22], [23], [24], Elliot-Kalton [15], [16], Fleming [18], Krasovskii-Subbotin [26]). These works are mainly dealing with unconstrained problems (except for [24]), and the value function is always continuous. Related problems, such as notions of local asymptotics, local and global stabilizations are studied in the article of Yong [37].

More recently, the techniques of viscosity solutions allow Evans \& Souganidis [17] to simplify the proofs of existence of a value and to characterize this value as unique solution of some Hamilton-Jacobi equation. Pursuit games without state constraints and for continuous value functions are studied by Soravia in [34]. Alziary [1] uses these methods for a particular continuous pursuit game with state-constraints (the Lion \& Man game).

In [32], Rozyev \& Subbotin prove the existence of a value for differential game without continuity and with some state-constraints for one player (Victor). However, these results couldn't directly be extended to differential games with separate dynamics and with state constraints on both dynamics. Actually, the basic idea of this method - the so-called "extremal aiming" (which gives the strategy) - is not applicable to situations where there are state constraints for both players. Let us also point out that their approach is devoted to games in a context of strategies slightly different from the one used in this paper. This method is adapted to the kind of strategies we use, the non-anticipative strategies, and to the viscosity solution approach, by Bardi, Bottacin and Falcone in [5]. Our Definition of value function is (partially) borrowed from this paper.

When this paper was complete, we received a preprint of Bardi, Koike \& Soravia [6] establishing the existence of a value for pursuit games with state constraints when this value is continuous. Let us point out that the constraints in [6] are more general than ours. However, the method developed in [6] heavily relies on the fact that the value function is continuous. For getting this continuity, the authors assume that one of the value functions vanishes at the boundary of the target and is continuous on this boundary, and that each control system 
(1) and (2) is locally controllable.

In this paper, we do not make any controllability condition at the boundary of the target, so that the value of the game is in general not continuous. However, following Soner [33], we make some restriction on the dynamics at the boundary of the state constraints. Under this restriction, we prove that the pursuit-evasion game has a value. Let us point out that most examples given in the "classical theory" of differential games are concerned by our result.

We follow here the method described in [13] for characterizing the value functions of differential game: Namely, we reduce the study of the pursuit game to a qualitative differential game called "approach-evasion game". The idea of reducing a quantitative game to a qualitative one comes back to Isaacs [25]. Here we use an idea of Frankowska for control problems, which amounts to characterize the epigraph of the value function (see [20] for instance, or [13] for further references on the subject). So, in a first step, we study this qualitative game (or game of kind, in Isaacs terminology), we give an "alternative result" for that game and characterize the victory domains in a geometric way. Then, in a second step, we interpret the pursuit game as a qualitative pursuit evasion game in order to prove that there is a value to the problem.

\section{Existence of a value for the pursuit game}

In this section, we state the main result of this paper, namely that pursuit games have a value. For doing so, we first introduce some notations and assumptions.

\section{$1.1 \quad$ Notations and assumptions}

The dynamics of the system are:

$$
\left\{\begin{array}{l}
y^{\prime}(t)=g(y(t), u(t)), u(t) \in U, y(t) \in K_{U} \\
z^{\prime}(t)=h(z(t), v(t)), v(t) \in V, z(t) \in K_{V}
\end{array}\right.
$$

with $y \in \boldsymbol{R}^{l}, z \in \boldsymbol{R}^{m}$. We set $x(t):=(y(t), z(t)), N:=l+m$ and

$$
f(x, u, v):=f(y, z, u, v):=\{g(y, u)\} \times\{h(z, v)\}
$$

The first player (Ursula), controlling $u$, has to ensure that $y(t) \in K_{U}$, while the second player (Victor), playing with $v$, has to ensure that $z(t) \in K_{V}$.

The sets $K_{U}$ and $K_{V}$ have smooth boundaries. Moreover, we assume some transversality conditions of the vector fields at the boundary of the state con- 
straints similar as that of Soner [33]. Namely,

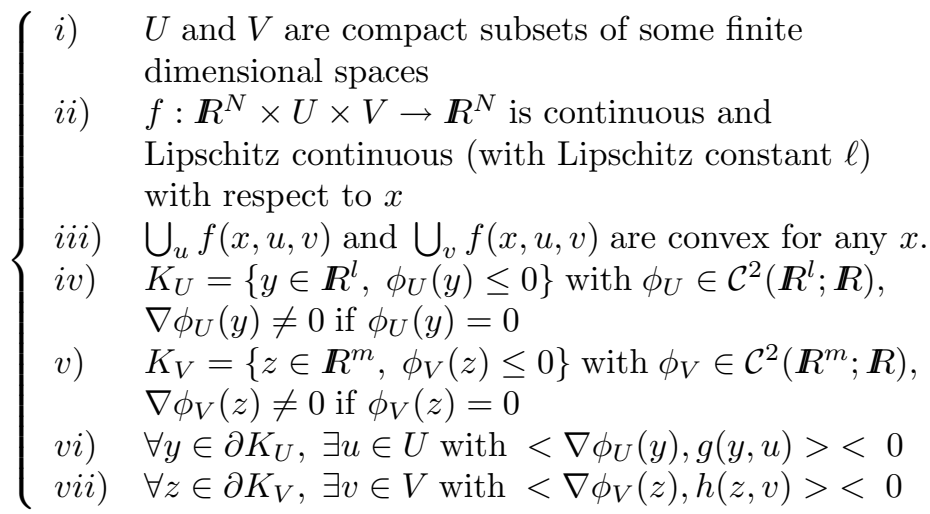

For any time-measurable controls $u(\cdot)$ and $v(\cdot)$, we denote by $y\left[y_{0}, u(\cdot)\right]$, $z\left[z_{0}, v(\cdot)\right]$ and $x\left[x_{0}, u(\cdot), v(\cdot)\right]$ the solutions (in the Caratheodory sense) to equations (1), (2) and to equation

$$
x^{\prime}(t)=f(x(t), u(t), v(t))
$$

starting respectively from $y_{0}, z_{0}$ and $x_{0}:=\left(y_{0}, z_{0}\right)$.

The sets of time measurable controls $u(\cdot): \boldsymbol{R}^{+} \rightarrow U$ and $v(\cdot): \boldsymbol{R}^{+} \rightarrow V$ are denoted respectively $\mathcal{U}$ and $\mathcal{V}$, while the sets of admissible controls are denoted by $\mathcal{U}\left(y_{0}\right)$ and $\mathcal{V}\left(z_{0}\right)$ :

$$
\left\{\begin{array}{l}
\mathcal{U}\left(y_{0}\right):=\left\{u(\cdot) \in \mathcal{U} \mid y\left[y_{0}, u(\cdot)\right](t) \in K_{U}, \forall t \geq 0\right\} \\
\mathcal{V}\left(z_{0}\right):=\left\{v(\cdot) \in \mathcal{V} \mid z\left[z_{0}, v(\cdot)\right](t) \in K_{V}, \forall t \geq 0\right\}
\end{array}\right.
$$

Under condition (3), it is well known (see [3]) that the sets $\mathcal{U}\left(y_{0}\right)$ and $\mathcal{V}\left(z_{0}\right)$ are not empty for any $y_{0} \in K_{U}$ and $z_{0} \in K_{V}$. Moreover, according to Arisawa-Lions [2] (see also [27]), the sets $\mathcal{U}\left(y_{0}\right)$ and $\mathcal{V}\left(z_{0}\right)$ are Lipschitz continuous with respect to $y_{0}$ and $z_{0}$. Namely (for instance for $y$ )

Lemma 1.1 Under assumption (3), for any positive constants $Q$ and $T$, there is some positive $\lambda=\lambda(Q, T, \ell)$ such that, for any $y_{0}, y_{1}$ belonging to $K_{U}$, with $\left\|y_{0}\right\| \leq Q$ and $\left\|y_{1}\right\| \leq Q$, for any admissible control $u_{0}(\cdot) \in \mathcal{U}\left(y_{0}\right)$, there is some admissible control $u_{1}(\cdot) \in \mathcal{U}\left(y_{1}\right)$ such that

$$
\forall t \in[0, T],\left\|y\left[y_{0}, u_{0}(\cdot)\right](t)-y\left[y_{1}, u_{1}(\cdot)\right](t)\right\| \leq\left\|y_{0}-y_{1}\right\| e^{\lambda t} .
$$

\section{Remarks on assumptions (3):}

1. The regularity assumptions on the domains $K_{U}$ and $K_{V}$ can also be weakened, by using an extension of Lemma 1.1 recently obtained by FrankowskaRampazzo in [21] for non smooth domains. The transversality condition has to be extended in a suitable way. The results of the present paper also hold true (with the exactly the same proof) under Frankowska-Rampazzo assumption provided that the sets $K_{U}$ and $K_{V}$ are sleek in the sense of [3]. 
2. Following also [21], the convexity assumption (iii) can be avoided. We have preferred not to do so for simplicity. However, if one omits this assumption, one has to modify the transversality assumption in a suitable way as well as the Definition of $\vartheta_{C}^{b}$ (see [21]).

The players play non-anticipative strategies. A map $\alpha: \mathcal{V}\left(z_{0}\right) \rightarrow \mathcal{U}\left(y_{0}\right)$ is a non-anticipative strategy (for the first player Ursula and for the point $x_{0}:=$ $\left.\left(y_{0}, z_{0}\right) \in K_{U} \times K_{V}\right)$ if, for any $\tau>0$ for any control $v_{1}(\cdot)$ and $v_{2}(\cdot)$ belonging to $\mathcal{V}\left(z_{0}\right)$, which coincide a.e. on $[0, \tau], \alpha\left(v_{1}(\cdot)\right)$ and $\alpha\left(v_{2}(\cdot)\right)$ coincide almost everywhere on $[0, \tau]$. We denote by $S_{U}\left(x_{0}\right)$ the set of such non-anticipative strategies for Ursula.

The non-anticipative strategies $\beta$ for the second player Victor are defined symmetrically and we denote by $S_{V}\left(x_{0}\right)$ the set of such strategies.

Throughout this paper, $B$ denotes the closed unit ball of $\boldsymbol{R}^{N}$ (endowed with the Euclidean norm) and $d_{S}(x)$ denotes the distance from a point $x$ to a set $S$. Moreover, if $S$ is a subset of $\boldsymbol{R}^{N}$ and $\varepsilon$ is positive, we denote by $S+\varepsilon B$ the set

$$
S+\varepsilon B=\left\{x \in \boldsymbol{R}^{N} \mid d_{S}(x) \leq \varepsilon\right\} .
$$

\subsection{The main Theorem}

Let $C \subset K_{U} \times K_{V}$ be a closed target. The hitting-time of $C$ for a trajectory $x(\cdot):=(y(\cdot), z(\cdot))$ is:

$$
\theta_{C}(x(\cdot)):=\min \{t \geq 0 \mid x(t) \in C\}
$$

If $x(t) \notin C$ for every $t \geq 0$, then we set $\theta_{C}(x(\cdot)):=+\infty$. In the pursuit game, Ursula wants to maximize $\theta_{C}$ while Victor wants to minimize it.

Definition 1.2 (Value functions) The lower optimal hitting time function is the map $\vartheta_{C}^{b}: K_{U} \times K_{V} \rightarrow \boldsymbol{R}^{+} \cup\{+\infty\}$ defined, for any $x_{0}:=\left(y_{0}, z_{0}\right)$, by

$$
\vartheta_{C}^{b}\left(x_{0}\right):=\inf _{\beta(\cdot) \in S_{V}\left(x_{0}\right)} \sup _{u(\cdot) \in \mathcal{U}\left(y_{0}\right)} \theta_{C}\left(x\left[x_{0}, u(\cdot), \beta(u(\cdot))\right]\right)
$$

The upper optimal hitting time function is the map $\vartheta_{C}^{\sharp}: K_{U} \times K_{V} \rightarrow \boldsymbol{R}^{+} \cup$ $\{+\infty\}$ defined, for any $x_{0}:=\left(y_{0}, z_{0}\right)$, by

$$
\vartheta_{C}^{\sharp}\left(x_{0}\right):=\lim _{\varepsilon \rightarrow 0^{+}} \sup _{\alpha(\cdot) \in S_{U}\left(x_{0}\right)} \inf _{v(\cdot) \in \mathcal{V}\left(z_{0}\right)} \theta_{C+\varepsilon B}\left(x\left[x_{0}, \alpha(v(\cdot)), v(\cdot)\right]\right)
$$

By convention, we set $\vartheta_{C}^{b}(x)=\vartheta_{C}^{\sharp}(x)=0$ on $C$.

\section{Remarks :}

1. Let us point out that the limit in the definition of $\vartheta_{C}^{\sharp}$ exists because the quantity

$$
\sup _{\alpha(\cdot) \in S_{U}\left(x_{0}\right)} \inf _{v(\cdot) \in \mathcal{V}\left(z_{0}\right)} \theta_{C+\varepsilon B}\left(x\left[x_{0}, \alpha(v(\cdot)), v(\cdot)\right]\right)
$$


is non decreasing with respect to $\varepsilon$. Such a Definition is used for instance in [5]. The meaning of such a definition is the following: Whatever strategy $\alpha$ is played, the second player can ensure the state of the system to go as close as he wants to the target before $\vartheta_{C}^{\sharp}\left(x_{0}\right)$ (but the state of the system need not reach the target).

2. The following Definition of the upper value function has been used in several papers:

$$
\widehat{\vartheta}_{C}^{\sharp}\left(x_{0}\right):=\sup _{\alpha(\cdot) \in S_{U}\left(x_{0}\right)} \inf _{v(\cdot) \in \mathcal{V}\left(z_{0}\right)} \theta_{C}\left(x\left[x_{0}, \alpha(v(\cdot)), v(\cdot)\right]\right)
$$

However, without controllability assumptions on the boundary of the target, we cannot hope to have a value with this Definition of upper value function. For instance, if one considers the unconstrained pursuit game where the dynamics is

$$
\left\{\begin{array}{l}
y^{\prime}(t)=u(t) \text { where } u(t) \in U=[-1,1] \\
z_{1}^{\prime}(t)=v(t) \text { where } v(t) \in V=[-1,1] \\
z_{2}^{\prime}(t)=1
\end{array}\right.
$$

and the target is $C=\left\{\left(y, z_{1}, z_{2}\right) \in \mathbb{R}^{3} \mid y=z_{1}\right.$ and $\left.z_{2}=1\right\}$, then

$$
\widehat{\vartheta}_{C}^{\sharp}(0,0,0)=+\infty
$$

while

$$
\vartheta_{C}^{b}(0,0,0)=\vartheta_{C}^{\sharp}(0,0,0)=1 .
$$

Proof: We first prove the last equality: Let us notice that $\beta(u(\cdot))(t)=$ $u(t)$ is an optimal strategy for the second player. Hence $\vartheta_{C}^{b}(0,0,0)=1$. Equality $\vartheta_{C}^{b}(0,0,0)=\vartheta_{C}^{\sharp}(0,0,0)$ comes from Theorem 1.3 below.

We now prove the first equality. We define the non-anticipative strategy $\alpha$ in the following way. For any control $v(\cdot)$, let $z_{1}(\cdot)$ be the solution to

$$
\left\{\begin{array}{l}
z_{1}^{\prime}(t)=v(t) \\
z_{1}(0)=0
\end{array}\right.
$$

We set $c=\liminf _{h \rightarrow 0^{+}} z_{1}(h) / h$. Since $v(t) \in[-1,1]$, we have $c \in[-1,1]$. If $c>-1$, we set $\alpha(v(\cdot))(t)=-1$ while, if $c=-1$, we set $\alpha(v(\cdot))(t)=1$. Let us point out that such a map $\alpha$ is a non-anticipative strategy. We claim that, for any control $v(\cdot)$, the solution $x(\cdot)=x[0, \alpha(v(\cdot)), v(\cdot)]$ never touches the target.

We consider two cases. If on the one hand, $c>-1$, then there is some $\tau \in(0,1)$ such that

$$
\forall t \in(0, \tau], z_{1}(t) \geq \frac{(c-1)}{2} t>-t=y(t)
$$


Hence

$$
\forall t \geq \tau, z_{1}(t) \geq \frac{(c-1)}{2} \tau-(t-\tau)>-t=y(t)
$$

Therefore, for any $t, z_{1}(t)>y(t)$, so that $x(t) \notin C$.

If, on another hand, $c=-1$, there is a sequence $t_{n} \rightarrow 0^{+}$such that $\lim _{n} z_{1}\left(t_{n}\right) / t_{n}=-1$. Hence there is some $n$ such that $t_{n} \in(0,1)$ and $z_{1}\left(t_{n}\right) \leq t_{n} / 2$. Then

$$
\forall t \geq t_{n}, z_{1}(t) \leq t_{n} / 2+\left(t-t_{n}\right)<t=y(t) .
$$

Therefore, for any $t \geq t_{n}, z_{1}(t)<y(t)$, so that $x(t) \notin C$.

3. A natural question is: What happens if one modifies the lower value function in the same way? We show below that

$$
\vartheta_{C}^{b}\left(x_{0}\right):=\lim _{\varepsilon \rightarrow 0^{+}} \inf _{(\cdot) \in S_{V}\left(x_{0}\right)} \sup _{u(\cdot) \in \mathcal{U}\left(y_{0}\right)} \theta_{C+\epsilon B}\left(x\left[x_{0}, u(\cdot), \beta(u(\cdot))\right]\right)
$$

(see Proposition 3.2).

4. A last remark, which is not really interesting for differential games, but maybe is interesting on a p.d.e. point of view. In the theory of viscosity solutions, one often characterizes the solution through its lower semicontinuous and upper semi-continuous envelope. In the above example we can notice that the lower semi-continuous envelope of $\widehat{\vartheta}_{C}^{\sharp}$ is not equal to $\vartheta_{C}^{\sharp}$.

Theorem 1.3 Assume that conditions (3) are fulfilled. Then the game has a value:

$$
\forall x_{0} \in K_{U} \times K_{V}, \vartheta_{C}^{b}\left(x_{0}\right)=\vartheta_{C}^{\sharp}\left(x_{0}\right)
$$

This Theorem is proved in section 4 by reducing the pursuit game to a qualitative differential game called the pursuit evasion game. We deduce from the "alternative Theorem" for this qualitative game (see Theorem 2.6 below) the existence of a value for the pursuit game.

Moreover the proof gives a geometric characterization of the value function. This characterization can be formulated as a Hamilton-Jacobi-Isaacs equation (see [13]). We shall not do so for sake of shortness. As indicated in [13], we can also derive from this characterization numerical schemes for computing the value function.

\section{An Alternative Theorem for a qualitative dif- ferential game with state constraints}

In this section, we study the differential game in which the first player Ursula, controlling system (1), aims the state of the full system at reaching an open 
target $\mathcal{O}$ while the other player, Victor, controlling system (2), aims the state of the system at avoiding $\mathcal{O}$ and - if possible - at reaching some given evasion set $\mathcal{E}$. This game is very close to the approach-evasion game of Krasovskii-Subbotin $[26]$.

\subsection{Statement of the qualitative problem}

Definition 2.1 Let $\mathcal{O} \subset K_{U} \times K_{V}$ be an open target and $\mathcal{E} \subset K_{U} \times K_{V}$ be a closed evasion set. The victory domains of the players are defined as follows:

- Victor's victory domain is the set of initial positions $x_{0}:=\left(y_{0}, z_{0}\right)$ belonging to $K_{U} \times K_{V}$ for which there is an admissible non-anticipative strategy $\beta: \mathcal{U}\left(y_{0}\right) \rightarrow \mathcal{V}\left(z_{0}\right)$ such that, for any admissible control $u(\cdot) \in \mathcal{U}\left(y_{0}\right)$, the solution $x\left[x_{0}, u(\cdot), \beta(u(\cdot))\right]$ avoids $\mathcal{O}$ as long as it does not reach $\mathcal{E}$ (or avoids $\mathcal{O}$ on $[0,+\infty)$ if it never reaches $\mathcal{E})$.

- Ursula's victory domain is the set of initial positions $x_{0}:=\left(y_{0}, z_{0}\right)$ belonging to $K_{U} \times K_{V}$ for which there are $T \geq 0, \varepsilon>0$ and an admissible non-anticipative strategy $\alpha: \mathcal{V}\left(z_{0}\right) \rightarrow \mathcal{U}\left(y_{0}\right)$ such that, for any admissible control $v(\cdot) \in \mathcal{V}\left(z_{0}\right)$, the solution $x\left[x_{0}, \alpha(v(\cdot)), v(\cdot)\right]$ reaches $\mathcal{O}_{\varepsilon}:=\left\{x \in \mathcal{O} \mid d_{\partial \mathcal{O}}(x)>\varepsilon\right\}$ at some time $\tau \leq T$ and does not reach $\mathcal{E}+\varepsilon B$ on $[0, \tau]$.

In this section, we prove the following alternative result: if $x$ belongs to $K_{U} \times K_{V}$, then $x$ belongs to one and only one victory domain. Moreover, we give a geometric characterization of the victory domains.

\subsection{The discriminating domains}

For $x:=(y, z) \in \mathbb{R}^{N}$, we set

$$
U(y):=\left\{u \in U \mid g(y, u) \in T_{K_{U}}(y)\right\}
$$

where $T_{K_{U}}(y)$ is the usual tangent half-space to the set with smooth boundary $K_{U}$ at $y$. Let us notice that, under assumptions (3), the set-valued map $y \rightarrow$ $f(y, U(y))$ is lower semi-continuous with convex compact values (see [4]).

Let us introduce the Hamiltonian of our system:

$$
H(x, p):= \begin{cases}\inf _{v \in V} \sup _{u \in U(y)}<f(x, u, v), p> & \text { if } x \notin \mathcal{E} \\ \min \left\{0, \inf _{v \in V} \sup _{u \in U(y)}<f(x, u, v), p>\right\} & \text { otherwise }\end{cases}
$$

where $x:=(y, z)$ and where

$$
\inf _{v \in V} \sup _{u \in U(y)}<f(x, u, v), p>=\sup _{u \in U(y)}<g(y, u), p_{y}>+\inf _{v \in V}<h(z, v), p_{z}>.
$$

Definition 2.2 A closed subset $D$ of $K_{U} \times K_{V}$ is a discriminating domain for $H$ if and only if,

$$
\forall x \in D, \forall p \in N P_{D}(x), H(x, p) \leq 0
$$


where $N P_{D}(x)$ denotes the set of proximal normal to $D$ at $x$, i.e., the set of $p \in \mathbb{R}^{N}$ such that the distance of $x+p$ to $D$ is equal to $\|p\|$.

For the original definition of discriminating domains, see Aubin [3].

Discriminating domains can be characterized in two different ways:

Theorem 2.3 Suppose that assumptions (3) are fulfilled. A closed subset D of $K_{U} \times K_{V}$ is a discriminating domain for $H$ if and only if, for any initial position $x_{0}=\left(y_{0}, z_{0}\right) \in D$, there is a non-anticipative strategy $\beta \in S_{V}\left(x_{0}\right)$, such that, for any $u(\cdot) \in \mathcal{U}\left(y_{0}\right)$, the solution $x\left[x_{0}, u(\cdot), \beta(u(\cdot))\right]$ remains in $D$ until it reaches $\mathcal{E}$ (or remains in $D$ on $[0,+\infty)$ if it never reaches $\mathcal{E}$ ).

Remark : This result was proved independently and in the same time by Plaskacz [29] and by the first author [10] when $K_{U}=\boldsymbol{R}^{l}$ and $\mathcal{E}=\emptyset$. For timemeasurable dynamics, see also [14]. Theorem 2.3 (in a more general form) was announced in [12].

Theorem 2.4 Suppose that assumptions (3) are fulfilled. A closed set $D \subset$ $K_{U} \times K_{V}$ is a discriminating domain for $H$ if and only if, for any initial position $x_{0}:=\left(y_{0}, z_{0}\right) \in D$, for any admissible non-anticipative strategy $\alpha: \mathcal{V}\left(z_{0}\right) \rightarrow$ $\mathcal{U}\left(y_{0}\right)$, for any $T \geq 0$ and any $\varepsilon>0$, there is an admissible control $v(\cdot) \in \mathcal{V}\left(z_{0}\right)$ such that the solution $x\left[x_{0}, \alpha(v(\cdot)), v(\cdot)\right]$ remains in $D+\varepsilon B$ on $[0, T]$ as long as it does not reach $\mathcal{E}+\varepsilon B$. Namely,

- either there is some $\tau \leq T$ such that $x\left[x_{0}, \alpha(v(\cdot)), v(\cdot)\right](\tau)$ belongs to $\mathcal{E}+\varepsilon B$ and $x\left[x_{0}, \alpha(v(\cdot)), v(\cdot)\right](t) \in D+\varepsilon B$ for $t \in[0, \tau]$,

- or $x\left[x_{0}, \alpha(v(\cdot)), v(\cdot)\right](t) \in D+\varepsilon B$ for $t \in[0, T]$.

Remark : Note that, if $D \subset\left(K_{U} \times K_{V}\right) \backslash \mathcal{O}$ is a discriminating domain, then $D$ is a subset of Victor's victory domain (according to Theorem 2.3) and has an empty intersection with Ursula's victory domain (according to Theorem 2.4).

The proof of Theorems 2.3 and 2.4, being rather technical, are given in appendix.

\subsection{The Alternative Theorem}

We now characterize Victor's and Ursula's victory domains. For that purpose, we first recall the definition of the discriminating kernel.

Proposition 2.5 ([11]) Let $H: \mathbb{R}^{N} \times \mathbb{R}^{N} \rightarrow \boldsymbol{R}$ be a lower semi-continuous map. If $K$ is a subset of $\boldsymbol{R}^{N}$, then $K$ contains a largest (for the inclusion) closed discriminating domain for $H$. This set is called the discriminating kernel of $K$ for $H$ and is denoted $\operatorname{Disc}_{H}(K)$.

Theorem 2.6 (Alternative Theorem) Let $H$ be defined by (5) and assume that (3) is fulfilled. Then,

- Victor's victory domain is equal to $\operatorname{Disc}_{H}(\mathcal{K})$.

- Ursula's victory domain is equal to $\left(K_{U} \times K_{V}\right) \backslash \operatorname{Disc}_{H}(\mathcal{K})$

where $\mathcal{K}:=\left(K_{U} \times K_{V}\right) \backslash \mathcal{O}$. 
In particular, any point of $\left(K_{U} \times K_{V}\right)$ belongs either to Victor's victory domain or to Ursula's one. When $K_{U}=K_{V}=\boldsymbol{R}^{N}$, this characterization can be found in [10].

The proof is given in appendix.

\section{Proof of the existence of a value for the pur- suit game}

We come back to the problem of the existence of a value (see section 1), i.e., to the equality between $\vartheta_{C}^{b}$ and $\vartheta_{C}^{\sharp}$. We are going to prove that their epigraph are equal. Let us recall that the epigraph of $\vartheta_{C}^{b}$ (for instance) is a subset of $\boldsymbol{R}^{N+1}$ defined by

$$
\mathcal{E} p i\left(\vartheta_{C}^{b}\right)=\left\{(x, \rho) \in \mathbb{R}^{N+1} \mid \vartheta_{C}^{b}(x) \leq \rho\right\}
$$

In the sequel, we always denote by $(x, \rho)$ any point of $\boldsymbol{R}^{N+1}$, where $x \in \mathbb{R}^{N}$ and $\rho \in \mathbb{R}$.

Theorem 3.1 Assume that conditions (3) are fulfilled. Then we have:

$$
\mathcal{E} p i\left(\vartheta_{C}^{b}\right)=\operatorname{Disc}_{H}(\mathcal{K})=\mathcal{E} p i\left(\vartheta_{C}^{\sharp}\right)
$$

where $\mathcal{K}:=K_{U} \times K_{V} \times \mathbb{R}^{+}$and where the Hamiltonian $H: \mathbb{R}^{N+1} \times \mathbb{R}^{N+1} \rightarrow \mathbb{R}$ is defined by:

$$
\begin{array}{ll}
\forall(x, \rho) \in \boldsymbol{R}^{N+1},\left(p_{x}, p_{\rho}\right) \in \mathbb{R}^{N+1}, & \\
H\left(x, \rho, p_{x}, p_{\rho}\right) & \\
\quad:=\sup _{u \in U(y)} \inf _{v \in V}<f(x, u, v), p_{x}>-p_{\rho} & \text { if } x \notin C \\
\quad:=\min \left\{0 ; \sup _{u \in U(y)} \inf _{v \in V}<f(x, u, v), p_{x}>-p_{\rho}\right\} & \text { otherwise }
\end{array}
$$

\section{Remarks :}

- This result proves Theorem 1.3 since the functions $\vartheta_{C}^{b}$ and $\vartheta_{C}^{\sharp}$, having the same hypograph, are equal.

- We can deduce from this result that the map $\vartheta_{C}^{b}=\vartheta_{C}^{\sharp}$ is lower semicontinuous, since its epigraph is closed (the set $\operatorname{Disc}_{H}(\mathcal{K})$ being closed from Proposition 2.5).

- We can also derive from the proof of Theorem 3.1 the existence of an optimal strategy for pursuer (Victor).

Proof of Theorem 3.1 :

Proof of the first equality of (6):

Let us introduce the following dynamic: $\tilde{f}: \boldsymbol{R}^{N} \times \mathbb{R} \times U \times V \rightarrow \boldsymbol{R}^{N} \times \mathbb{R}$ given by

$$
\widetilde{f}(x, \rho, u, v):=\{f(x, u, v)\} \times\{-1\}
$$


Note that the Hamiltonian $H$ defined above is actually of the form of the Hamiltonian $H$ defined by (5) for the dynamics $\widetilde{f}$ and for the closed evasion set defined by $\mathcal{E}:=C \times \mathbb{R}$.

From Theorem 2.6, the discriminating kernel of $\mathcal{K}$ for $\widetilde{H}$ is the set of initial positions $\left(x_{0}, \rho_{0}\right) \in \mathcal{K}$ for which there is some non-anticipative strategy $\beta \in$ $S_{V}\left(x_{0}\right)$ for Victor such that, for any $u(\cdot) \in \mathcal{U}\left(y_{0}\right)$, the solution to

$$
\left\{\begin{array}{l}
x^{\prime}(t)=f(x(t), u(t), \beta(u(\cdot))(t)) \\
\rho^{\prime}(t)=-1 \\
x(0)=x_{0} \quad \& \quad \rho(0)=\rho_{0}
\end{array}\right.
$$

remains in $\mathcal{K}$ until it reaches the set $\mathcal{E}$.

Let $\left(x_{0}, \rho_{0}\right)$ belong to $\operatorname{Disc}_{\widetilde{H}}(\mathcal{K}), \beta$ be an associated non-anticipative strategy and $(x(\cdot), \rho(\cdot))$ be the solution to $(7)$. Since the solution $(x(\cdot), \rho(\cdot))$ remains in $\mathcal{K}$ as long as it does not reach $\mathcal{E}, \rho(t)=\rho_{0}-t \geq 0$ as long as $x(t) \notin C$. Thus the solution $x\left[x_{0}, u(\cdot), \beta(u(\cdot))\right]$ reaches $C$ before $\rho_{0}$. In particular,

$$
\vartheta_{C}^{b}\left(x_{0}\right) \leq \sup _{u(\cdot) \in \mathcal{U}} \theta_{C}\left(x\left[x_{0}, u(\cdot), \beta(u(\cdot))\right]\right) \leq \rho_{0} .
$$

So $\left(x_{0}, \rho_{0}\right)$ belongs to $\mathcal{E} p i\left(\vartheta_{C}^{b}(\cdot)\right)$ and $\operatorname{Disc}_{\widetilde{H}}(\mathcal{K}) \subset \mathcal{E} p i\left(\vartheta_{C}^{b}(\cdot)\right)$.

For proving the converse inclusion, let $x_{0}$ belong to the domain of $\vartheta_{C}^{b}(\cdot)$ and let $\rho_{0}>\vartheta_{C}^{b}\left(x_{0}\right)$. There is some non-anticipative strategy $\beta$ for Victor such that, for any $u(\cdot) \in \mathcal{U}\left(y_{0}\right)$, the solution $x\left[x_{0}, u(\cdot), \beta(u(\cdot))\right]$ reaches $C$ before $\rho_{0}$. In particular, the solution $(x(\cdot), \rho(\cdot))$ to $(7)$ remains in $\mathcal{K}$ until it reaches $\mathcal{E}$, so that $\left(x_{0}, \rho_{0}\right)$ belongs to $\operatorname{Disc}_{\widetilde{H}}(\mathcal{K})$ from Theorem 2.6. This holds true for any $\rho_{0}>\vartheta_{C}^{b}\left(x_{0}\right)$.

Since the discriminating kernel is a closed set, we have proved that

$$
\mathcal{E}_{\operatorname{pi}}\left(\vartheta_{C}^{b}(\cdot)\right) \subset \operatorname{Disc}_{\widetilde{H}}(\mathcal{K}) .
$$

Proof of the second equality of (6): Let $\left(y_{0}, z_{0}, w_{0}\right)$ belong to $\operatorname{Disc}_{\widetilde{H}}(\mathcal{K})$. Fix $\varepsilon>0$ and let $\alpha: \mathcal{V}\left(z_{0}\right) \rightarrow \mathcal{U}\left(y_{0}\right)$ be such that

$$
\forall v(\cdot) \in \mathcal{V}\left(z_{0}\right), \theta_{C+\varepsilon B}\left(x\left[x_{0}, \alpha(v(\cdot)), v(\cdot)\right]\right) \geq \vartheta_{C}^{\sharp}\left(y_{0}, z_{0}\right)-\varepsilon .
$$

From Theorem 2.6, for this non-anticipative strategy $\alpha$, for this $\varepsilon>0$ and for $T:=w_{0}+\varepsilon$, there is a control $v(\cdot) \in \mathcal{V}\left(z_{0}\right)$ such that the solution to

$$
\left\{\begin{array}{l}
x^{\prime}(t)=f(x(t), \alpha(v(\cdot))(t), v(t)) \\
w^{\prime}(t)=-1 \\
y(0)=y_{0}, z(0)=z_{0}, w(0)=w_{0}
\end{array}\right.
$$

remains in $\mathcal{K}+\varepsilon B$ on $[0, T]$ as long as it does not reach $(C \times \mathbb{R})+\varepsilon B$.

Set $\tau:=\inf \left\{\theta_{C+\varepsilon B}((y(\cdot), z(\cdot))) ; T\right\}$. Then $w(t)=w_{0}-t$ on $[0, \tau]$ and $w(t) \geq$ $-\varepsilon$ for any $t \in[0, \tau]$. So $\tau \leq w_{0}+\varepsilon$. 
So finally, $w_{0} \geq \vartheta_{C}^{\sharp}\left(y_{0}, z_{0}\right)-2 \varepsilon$. Since this holds true for any $\varepsilon>0$, we have finally proved that $w_{0} \geq \vartheta_{C}^{\sharp}\left(y_{0}, z_{0}\right)$. Thus $\operatorname{Disc}_{\widetilde{H}}(\mathcal{K})$ is a subset of $\mathcal{E} p i\left(\vartheta_{C}^{\sharp}\right)$.

Conversely, let $w_{0}>\vartheta_{C}^{\sharp}\left(y_{0}, z_{0}\right)$. Then, for any non-anticipative strategy $\alpha: \mathcal{V}\left(z_{0}\right) \rightarrow \mathcal{U}\left(y_{0}\right)$, for any positive $\varepsilon$, there is a control $v(\cdot) \in \mathcal{V}\left(z_{0}\right)$ such that

$$
\theta_{C+\varepsilon B}\left(x\left[x_{0}, \alpha(v(\cdot)), v(\cdot)\right]\right) \leq w_{0}
$$

Note that the function $\left.t \rightarrow\left(x\left[x_{0}, \alpha(v(\cdot)), v(\cdot)\right)\right](t), w_{0}-t\right)$ is a solution to $(9)$. Moreover, this solution remains in $\mathcal{K}$ as long as the solution does not reach $\mathcal{E}+\varepsilon B$ (i.e., on $\left.\left[0, \theta_{C+\varepsilon B}\left(x\left[x_{0}, \alpha(v(\cdot)), v(\cdot)\right]\right)\right]\right)$. So $\left(y_{0}, z_{0}, w_{0}\right)$ belongs to $\operatorname{Disc}_{\widetilde{H}}(\mathcal{K})$. Since $\operatorname{Disc}_{\widetilde{H}}(\mathcal{K})$ is closed, this holds true for any $w_{0} \geq \vartheta_{C}^{\sharp}\left(y_{0}, z_{0}\right)$.

This proves the equality between $\operatorname{Disc}_{\widetilde{H}}(\mathcal{K})$ and $\mathcal{E} p i\left(\vartheta_{C}^{\sharp}\right)$.

We can also use the previous Theorem in order to prove some stability results. Namely

Proposition 3.2 Under the assumptions of Theorem 3.1

$$
\vartheta_{C}^{b}\left(x_{0}\right):=\lim _{\varepsilon \rightarrow 0} \inf _{\beta(\cdot) \in S_{V}\left(x_{0}\right)} \sup _{u(\cdot) \in \mathcal{U}\left(y_{0}\right)} \theta_{C+\epsilon B}\left(x\left[x_{0}, u(\cdot), \beta(u(\cdot))\right]\right)
$$

Proof : Let us denote by $\vartheta_{C+\epsilon B}^{b}$ and $H_{\epsilon}$ the value and the Hamiltonian associated with the target $C+\epsilon B$ :

$$
\begin{aligned}
& \forall(x, \rho) \in \mathbb{R}^{N+1},\left(p_{x}, p_{\rho}\right) \in \boldsymbol{R}^{N+1}, \\
& H\left(x, \rho, p_{x}, p_{\rho}\right) \\
& :=\sup _{u \in U(y)} \inf _{v \in V}<f(x, u, v), p_{x}>-p_{\rho} \\
& :=\min \left\{0 ; \sup _{u \in U(y)} \inf _{v \in V}<f(x, u, v), p_{x}>-p_{\rho}\right\} \\
& \text { if } x \notin C+\epsilon B \\
& \text { otherwise }
\end{aligned}
$$

According to Theorem 3.1, we have:

$$
\mathcal{E} p i\left(\vartheta_{C+\epsilon B}^{b}\right)=\operatorname{Disc}_{H_{\epsilon}}(\mathcal{K})
$$

where $\mathcal{K}:=K_{U} \times K_{V} \times \boldsymbol{R}^{+}$. Since $H_{\epsilon} \leq H$, the following inequality is obvious:

$$
\operatorname{Disc}_{H}(\mathcal{K}) \subset \operatorname{Disc}_{H_{\epsilon}}(\mathcal{K}) .
$$

Conversely, since the lower semi-continuous Hamiltonians $H_{\epsilon}$ converge in the sense of Proposition 1.2 of [11], this Proposition states that the decreasing limit of $\operatorname{Disc}_{H_{\epsilon}}(\mathcal{K})$ is a discriminating domain for $H$. Since this limit is contained in $\mathcal{K}$, because so are $\operatorname{Disc}_{H_{\epsilon}}(\mathcal{K})$, it is contained in $\operatorname{Disc}_{H}(\mathcal{K})$. So we have proved that

$$
\bigcap_{\epsilon>0} \operatorname{Disc}_{H_{\epsilon}}(\mathcal{K})=\operatorname{Disc}_{H}(\mathcal{K}),
$$

which is equivalent with saying that

$$
\vartheta_{C}^{b}\left(x_{0}\right):=\lim _{\epsilon \rightarrow 0^{+}} \inf _{\beta(\cdot) \in S_{V}\left(x_{0}\right)} \sup _{u(\cdot) \in \mathcal{U}\left(y_{0}\right)} \theta_{C+\epsilon B}\left(x\left[x_{0}, u(\cdot), \beta(u(\cdot))\right]\right) .
$$




\section{Appendix}

We now prove Theorems 2.3, 2.4 and 2.6. The proof of these results has the same framework as the proof of Theorems 2.1, 2.2, 2.3 and 2.4 of [10]. However, the key points of the proofs essentially differ because of the presence of the constraints. Hence, for sake of shortness, we refer to [10] for the framework of the proofs and we only give the key points.

\subsection{Proof of Theorem 2.3}

The condition is sufficient : Assume that $D$ is a discriminating domain. The crucial point of the proof is the following Lemma:

Lemma 4.1 Suppose that assumptions of Theorem 2.3 are fulfilled and assume that $D$ is a discriminating domain for $H$. Then for any $x_{0}=\left(y_{0}, z_{0}\right) \in D$, for any control $u(\cdot) \in \mathcal{U}\left(y_{0}\right)$, there is a control $v(\cdot) \in \mathcal{V}\left(z_{0}\right)$ such that the solution $x(\cdot):=x\left[x_{0}, u(\cdot), v(\cdot)\right]$ remains in $D$ until it reaches $\mathcal{E}$ (or remains in $D$ forever if it does not reach $\mathcal{E}$ ).

The sequel of the proof runs as in Theorem 2.1 of [10] or as in [14].

Proof of Lemma 4.1 : Let us assume that $D$ is a discriminating domain for $H$. Let $x_{0} \in D$ and $u_{0}(\cdot) \in \mathcal{U}\left(y_{0}\right)$. It is enough to prove that there is a measurable control $v(\cdot) \in \mathcal{V}$ and a time $T>0$ such that $x(\cdot):=x\left[x_{0}, u_{0}(\cdot), v(\cdot)\right]$ remains in $D$ on $[0, T]$ until it reaches $\mathcal{E}$. Let us notice that, if $x_{0} \in \mathcal{E}$, then the proof is obvious. We now assume $x_{0} \notin \mathcal{E}$.

We divide the proof in two steps: in the first step we assume that $y_{0}$ belongs to the interior of $K_{U}$ and in the second step that $y_{0}$ belongs to $\partial K_{U}$.

First step : We assume that there is some $T>0$ such that the solution $y(\cdot):=y\left[y_{0}, u_{0}(\cdot)\right]$ remains in $\operatorname{Int}\left(K_{U}\right)$ on $[0, T]$. Then we are going to prove that there is a control $v(\cdot) \in \mathcal{V}\left(z_{0}\right)$ such that $x\left[x_{0}, u(\cdot), v(\cdot)\right]$ remains in $D$ on $[0, T]$.

Let $\varepsilon>0$ be such that

$$
\forall t \in[0, T], y(t)+\varepsilon B \subset \operatorname{Int}\left(K_{U}\right) .
$$

Let us introduce the following open set:

$$
W:=\left\{(y, z) \in \boldsymbol{R}^{N} \mid \exists t \in[0, T],\|y(t)-y\|<\varepsilon\right\}=(y([0, T])+\varepsilon \stackrel{o}{B}) \times \mathbb{R}^{m} .
$$

Let us define the set-valued map:

$$
F(t, x):= \begin{cases}\bigcup_{v} \in V\left(x, u_{0}(t), v\right) & \text { if } x \notin \mathcal{E} \\ \overline{C o}\left\{\{0\} \cup \bigcup_{v \in V} f\left(x, u_{0}(t), v\right)\right\} & \text { if } x \in \mathcal{E}\end{cases}
$$

The main point of the proof is to check the assumptions for applying the measurable viability theorem of [20] for the set $W \cap D$ and $F$. 
Let us notice that $F$ is measurable and upper semi-continuous with respect to $x$. Moreover, $F$ has convex compact values. We claim that the set $W \cap D$ is a locally compact viability domain for $F$. Indeed, if $x:=(y, z) \in W \cap D$, then $y \in \operatorname{Int}\left(K_{U}\right)$, so that $U(y)=U$. Since $D$ is a discriminating domain and $U(y)=U$ on $W$ and $f(x, u, V)$ is convex, the proximal normal condition can be replaced by the following one (see for instance [11]):

$$
\forall x \in D \cap W, \forall u \in U, \exists v \in V \text { with } f(x, u, v) \in T_{D}(x) .
$$

Thus

$$
F(t, x) \cap T_{D}(x) \neq \emptyset \quad \text { a.e. } t \in[0, T],
$$

where $T_{D}(x)=\left\{v \in \mathbb{R}^{N}, \liminf _{h \rightarrow 0^{+}} d_{D}(x+h v) / h=0\right\}$. So we have proved that $W \cap D$ is a locally compact viability domain for $F$ on $[0, T]$.

The measurable viability theorem of [20] states that there is a solution $\bar{x}(\cdot)$ to the differential inclusion

$$
\left\{\begin{array}{l}
\bar{x}^{\prime}(t) \in F(t, \bar{x}(t)) \\
\bar{x}(0)=x_{0}
\end{array}\right.
$$

which remains in $D$ as long as it belongs to $W$. Note that, $\bar{x}(t)=(\bar{y}(t), \bar{z}(t))$ where $\bar{y}(\cdot)=y\left[y_{0}, u_{0}(\cdot)\right]=y(\cdot)$.

In particular, $\bar{x}(t)$ remains in $W$ on $[0, T]$, and thus also in $D$ on $[0, T]$. As long as $\bar{x}(\cdot)$ does not belong to $\mathcal{E}, \bar{x}(\cdot)$ is the solution to

$$
\bar{x}^{\prime}(t) \in \bigcup_{v} f\left(\bar{x}(t), u_{0}(t), v\right) .
$$

Then the Measurable Selection Theorem (Theorem 8.3.1 of [4]) states that there is some control $v(\cdot)$ such that the solution $x\left[x_{0}, u_{0}(\cdot), v(\cdot)\right]$ is equal to $\bar{x}(\cdot)$ until the solution reaches $\mathcal{E}$. When the solution has reached $\mathcal{E}$, we can set $v(t)=\bar{v}$ where $\bar{v}$ is any element of $V$. So we have finally defined a control $v(\cdot)$ such that the solution $x\left[x_{0}, u_{0}(\cdot), v(\cdot)\right]$ remains in $D$ on $[0, T]$ until it reaches $\mathcal{E}$.

Second step : Let us now assume that $y_{0}$ belongs to $\partial K_{U}$. From assumption (3), there is some $\bar{u} \in U\left(y_{0}\right)$ such that $w:=g\left(y_{0}, \bar{u}\right)$ belongs to $\operatorname{Int}\left(T_{K_{U}}\left(y_{0}\right)\right)$.

Fix $\theta>0$ and define $x_{\theta}=\left(y_{0}+\theta w, z_{0}\right)$ and $y_{\theta}(\cdot):=y\left[y_{0}+\theta w, u_{0}(\cdot)\right]$. Since $K_{U}$ is smooth, the solution $y_{\theta}(\cdot)$ remains in $\operatorname{Int}\left(K_{U}\right)$ on some interval $[0, T]$ (with $T>0$ independent of $\theta$ ) for any $\theta$ sufficiently small.

Thanks to the first step, we know that there is some control $v_{\theta}(\cdot)$ such that the solution $x_{\theta}(\cdot):=x\left[x_{\theta}, u_{0}(\cdot), v_{\theta}(\cdot)\right]$ remains in $D$ on $[0, T]$ until it reaches $\mathcal{E}$. Since the set-valued map $\bigcup_{v \in V} f\left(x, u_{0}(t), v\right)$ is measurable, upper semicontinuous with respect to $x$ and has convex compact values, and since $\mathcal{E}$ is closed, there are a sequence $\theta_{n} \rightarrow 0^{+}$and a sequence $x_{\theta_{n}}(\cdot)$ which converges to some $x(\cdot)$ starting from $x_{0}$ and remaining in $D$ on $[0, T]$ until it reaches $\mathcal{E}$. Since, as long as the $x(\cdot)$ has not reached $\mathcal{E}, x(\cdot)$ is a solution to

$$
x^{\prime}(t) \in \bigcup_{v} f\left(x(t), u_{0}(t), v\right),
$$


the Measurable Selection Theorem states that there exists a control $v(\cdot) \in \mathcal{V}\left(z_{0}\right)$ such that $x(\cdot)=x\left[x_{0}, u_{0}(\cdot), v(\cdot)\right]$ as long as this solution has not reached $\mathcal{E}$. After the solution has reached $\mathcal{E}$, we can set $v(t)=\bar{v}$ where $\bar{v}$ is any element of $V$.

The condition is necessary : Assume that the closed set $D$ satisfies the $\overline{\text { property given in Theorem 2.3. }}$ Let $\bar{x}=(\bar{y}, \bar{z}) \in D \backslash \mathcal{E}, p \in N P_{D}(\bar{x})$ and $\bar{u} \in U(\bar{y})$. We have to prove that

$$
\sup _{u \in U(\bar{y})} \inf _{v \in V}<f(\bar{x}, u, v), p>\leq 0 .
$$

Since the set-valued map $y \rightarrow g(y, U(y))$ is lower semi-continuous with compact convex values, Michael Selection Theorem (see [4]) yields the existence of a continuous selection $\widetilde{w}: K_{U} \rightarrow U$ of this set-valued map, i.e., $\widetilde{w}(y) \in g(y, U(y))$ such that $\widetilde{w}(\bar{y})=g(\bar{y}, \bar{u})$. Let $y(\cdot)$ be any solution to the differential equation

$$
\left\{\begin{array}{l}
y^{\prime}(t)=\widetilde{w}(y(t)), y(t) \in K_{U} \\
y(0)=\bar{y}
\end{array}\right.
$$

Then from the Measurable Selection Theorem, there is some measurable control $u(\cdot) \in \mathcal{U}(\bar{y})$ with $y(t)=y[\bar{y}, u(\cdot)](t)$. Note that $y^{\prime}(0)=g(\bar{y}, \bar{u})$.

Let $\beta$ be a non-anticipative strategy as in Theorem 2.3. Then $x(\cdot):=$ $x[\bar{x}, u(\cdot), \beta(u(\cdot))]$ remains in $D$ and thus in $\overline{R^{N} \backslash(\bar{x}+p+\|p\| B)}$. Then from standard arguments, there is a sequence $t_{k} \rightarrow 0^{+}$such that $\left(x\left(t_{k}\right)-\bar{x}\right) / t_{k}$ converges to some $v=\left(v_{y}, v_{z}\right)$ with $<v, p>\leq 0$. From the very construction of $u(\cdot), v_{y}=g(\bar{y}, \bar{u})$. Since $h(z, V)$ is convex, $v_{z} \in h(\bar{z}, V)$. Thus

$$
\sup _{u \in U(\bar{y})} \inf _{v \in V}<f(\bar{x}, u, v), p>\leq 0 .
$$

\subsection{Proof of Theorem 2.4}

The condition is sufficient:

The proof of the sufficient condition follows the proof of Theorem 2.3 in [10] with Lemma 4.2 below instead of Lemma 4.4 of [10]. In Lemma 4.4 of [10], we use a kind of "extremal aiming" method. Extremal aiming [26] amounts to associate with any point $x \notin D$ some projection $\bar{x}$ of $x$ onto $D$, and to play, for Ursula some $u \in U$ such that $\inf _{v}<f(\bar{x}, u, v), x-\bar{x}>$ is maximum, and for Victor some $v \in V$ such that $\sup _{u}<f(\bar{x}, u, v), x-\bar{x}>$ is minimum. Unfortunately, this method fails here since the players have to play admissible strategies and the strategies given by the extremal aiming method have no reason to be admissible.

For stating Lemma 4.2, let us fix some notations. Since we are only working on the bounded interval $[0, T]$ and with solutions starting from initial position $x_{0}$, we denote by $Q$ a radius such that any solution starting from $x_{0}$ remains in $Q B$ on $[0, T]$. We denote by $M$ a upper bound of $\|f\|$ on $Q B$. 
Lemma 4.2 Under the assumptions of Theorem 2.4, there are positive constants (depending on $Q, T, M$ and $\ell) a$ and $b$ such that, for any $x \in\left(K_{U} \times\right.$ $\left.K_{V}\right) \cap Q B$, with $x \notin D$ but $d_{D}(x) \leq Q$, for any admissible non-anticipative strategy $\alpha(\cdot) \in S_{U}(x)$, for any $\tau \in[0, T]$, there is some admissible control $v(\cdot) \in \mathcal{V}(z)$ such that

$$
d_{D}^{2}(x[x, \alpha(v(\cdot)), v(\cdot)](\tau)) \leq d_{D}^{2}(x)[1+a \tau]+b \tau^{2}
$$

Proof of Lemma 4.2 : Let $\bar{x}:=(\bar{y}, \bar{z})$ belong to the projection of $x$ onto $D$. We also set $p=\left(p_{y}, p_{z}\right)=x-\bar{x}$. Recall that $p$ belongs to $N P_{D}(\bar{x})$ and $\|p\| \leq Q$. Let us fix $\tau \in(0, T]$.

Let us consider $\widetilde{u}(\cdot) \in \mathcal{U}(y)$ an admissible control such that

$$
<y[y, \widetilde{u}(\cdot)](\tau), p_{y}>=\max _{u(\cdot) \in \mathcal{U}(y)}<y[y, u(\cdot)](\tau), p_{y}>.
$$

Thanks to Lemma 1.1, there is some control $\bar{u}(\cdot) \in \mathcal{U}(\bar{y})$ such that

$$
\|y[\bar{y}, \bar{u}(\cdot)](\tau)-y[y, \widetilde{u}(\cdot)](\tau)\|^{2} \leq\left\|p_{y}\right\|^{2} e^{2 \lambda \tau} .
$$

From Lemma 4.1, there is some admissible control $\bar{v}(\cdot) \in \mathcal{V}(\bar{z})$ such that the solution $x[\bar{x}, \bar{u}(\cdot), \bar{v}(\cdot)]$ remains in $D$. Thanks to Lemma 1.1, there is some control $v(\cdot) \in \mathcal{V}(z)$ such that

$$
\|z[\bar{z}, \bar{v}(\cdot)](\tau)-z[z, v(\cdot)](\tau)\|^{2} \leq\left\|p_{z}\right\|^{2} e^{2 \lambda \tau}
$$

Set $u(\cdot):=\alpha(v(\cdot))$. Then

$$
\begin{aligned}
& d_{D}^{2}(x[x, u(\cdot), v(\cdot)](\tau)) \\
& \quad \leq\|x[\bar{x}, \bar{u}(\cdot), \bar{v}(\cdot)](\tau)-x[x, u(\cdot), v(\cdot)](\tau)\|^{2} \\
& \quad \leq\left\|p_{z}\right\|^{2} e^{2 \lambda \tau}+\|y[\bar{y}, \bar{u}(\cdot)](\tau)-y[y, u(\cdot)](\tau)\|^{2} \\
& \quad \leq(1+a \tau)\left\|p_{z}\right\|^{2}+\|y[\bar{y}, \bar{u}(\cdot)](\tau)-y[y, u(\cdot)](\tau)\|^{2}
\end{aligned}
$$

If we set $\bar{y}(\cdot):=y[\bar{y}, \bar{u}(\cdot)], y(\cdot):=y[y, u(\cdot)]$, and $\widetilde{y}(\cdot):=y[y, \widetilde{u}(\cdot)]$, we have

$$
\begin{aligned}
& \|\bar{y}(\tau)-y(\tau)\|^{2} \\
& \quad=\|\bar{y}(\tau)-\widetilde{y}(\tau)\|^{2}+\|\widetilde{y}(\tau)-y(\tau)\|^{2}+2<\bar{y}(\tau)-\widetilde{y}(\tau) ; \widetilde{y}(\tau)-y(\tau)> \\
& \quad \leq\left\|p_{y}\right\|^{2} e^{2 \lambda \tau}+4 M^{2} \tau^{2}+2<-p_{y} ; \widetilde{y}(\tau)-y(\tau)>+4 M^{2} \tau^{2} \\
& \quad \leq(1+a \tau)\left\|p_{y}\right\|^{2}+b \tau^{2}
\end{aligned}
$$

for some constants $a$ and $b$, since

$$
<p_{y} ; y[y, \widetilde{u}(\cdot)](\tau)>\geq<p_{y} ; y[y, u(\cdot)](\tau)>
$$

from the very construction of $\widetilde{u}(\cdot)$. Therefore

$$
d_{D}^{2}(x[x, u(\cdot), v(\cdot)](\tau)) \leq(1+a \tau)\|p\|^{2}+b \tau^{2}
$$

which is the desired result since $\|p\|=d_{D}(x)$. 
Necessary condition : let us assume that $D$ is not a discriminating domain for $H$. We are going to prove the existence of some point $x_{0}$ and of a nonanticipative strategy $\alpha: \mathcal{V}\left(z_{0}\right) \rightarrow \mathcal{U}\left(y_{0}\right)$, of positive $\varepsilon$ and $T$ such that, for any $v(\cdot) \in \mathcal{V}\left(z_{0}\right)$, the solution $x\left[x_{0}, \alpha(v(\cdot)), v(\cdot)\right]$ leaves $D+\varepsilon B$ before $T$ and avoids $\mathcal{E}+\varepsilon B$ on $[0, T]$.

Let $x_{0}=\left(y_{0}, z_{0}\right) \in \partial D$ be such that the normal condition is not satisfied. Clearly $x_{0} \notin \mathcal{E}$ and there is some $p=\left(p_{y}, p_{z}\right) \in N P_{D}\left(x_{0}\right)$ and some $\gamma>0$ such that

$$
\inf _{v \in V}<h\left(z_{0}, v\right), p_{z}>+\sup _{u \in U\left(y_{0}\right)}<g\left(y_{0}, u\right), p_{y}>\geq \gamma .
$$

Let $\bar{u} \in U\left(y_{0}\right)$ which achieves the maximum of $\left\langle g\left(y_{0}, u\right), p_{y}\right\rangle$. Then, since $y \rightarrow g(y, U(y))$ is lower semi-continuous with convex compact values, there is a continuous selection $\widetilde{g}(y)$ of $g(y, U(y))$ such that $\widetilde{g}\left(y_{0}\right)=g\left(y_{0}, \bar{u}\right)$ (by Michael Selection Theorem in [4] for instance).

Let $y(\cdot)$ be any solution to $y^{\prime}(t)=\widetilde{g}(y(t)), y(0)=y_{0}, y(t) \in K_{U}$ for any $t \geq 0$ (Nagumo Theorem [3] states that such a solution exists). There is some control $u(\cdot)$ such that $y(\cdot)=y\left[y_{0}, u(\cdot)\right]$ and $u(\cdot) \in \mathcal{U}\left(y_{0}\right)$ since $y(t) \in K_{U}$ for $t \geq 0$. Moreover, $y^{\prime}(0)=g\left(y_{0}, \bar{u}\right)$. In particular, there is some $\tau>0$ such that,

$$
\forall t \in(0, \tau),<y(t)-y_{0}, p_{y}>\geq t \max _{u \in U\left(y_{0}\right)}<g\left(y_{0}, u\right), p_{y}>-t \gamma / 3 .
$$

For $\tau>0$ sufficiently small, for any admissible control $v(\cdot) \in \mathcal{V}\left(z_{0}\right)$, we have

$$
\forall t \in(0, \tau),<z\left[z_{0}, v(\cdot)\right]-z_{0}, p_{z}>\geq t \inf _{v \in V\left(z_{0}\right)}<h\left(z_{0}, v\right), p_{z}>-t \gamma / 3 .
$$

Let $v(\cdot) \in \mathcal{V}\left(z_{0}\right)$ be any admissible control. Then, since $p$ is a proximal normal to $D$ at $x_{0}$, for any $t \in(0, \tau)$,

$$
d_{D}\left(x\left[x_{0}, u(\cdot), v(\cdot)\right](t)\right) \geq\|p\|-\left\|x\left[x_{0}, u(\cdot), v(\cdot)\right](t)-x_{0}-p\right\|
$$

Note that

$$
\begin{aligned}
& \left\|x\left[x_{0}, u(\cdot), v(\cdot)\right](t)-x_{0}-p\right\|^{2} \leq \\
& \|p\|^{2}-2<y\left[y_{0}, u(\cdot)\right](t)-y_{0}, p_{y}>-2<z\left[z_{0}, v(\cdot)\right](t)-z_{0}, p_{z}>+C t^{2} \leq \\
& \|p\|^{2}-2 t(\gamma-2 \gamma / 3)+C t^{2}
\end{aligned}
$$

for some constant $C$. So

$$
d_{D}\left(x\left[x_{0}, u(\cdot), v(\cdot)\right](t)\right) \geq\|p\|-\left[\|p\|^{2}-2 t \gamma / 3+C t^{2}\right]^{1 / 2}
$$

which is positive for any $t \in(0, \tau)$ if $\tau>0$ is sufficiently small.

In conclusion, the desired non-anticipative strategy $\alpha: \mathcal{V}\left(z_{0}\right) \rightarrow \mathcal{U}\left(y_{0}\right)$ is the constant map $\alpha(v(\cdot))=u(\cdot)$. 


\subsection{Proof of Theorem 2.6}

We first prove that $\operatorname{Disc}_{H}(\mathcal{K})$ is equal to Victor's victory domain. This runs as in the proof of Theorem 2.2 of [10] where we use the following sequence $K_{n}$ instead of the sequence $K_{n}$ of [10]:

$$
\left\{\begin{array}{ll}
K_{0}=\mathcal{K} & \\
K_{n+1}:=\{u(\cdot) \in \mathcal{U}(y), \exists v(\cdot) \in \mathcal{V}(z), \exists \tau \in[0,+\infty] \\
x=(y, z) \in K_{n} \mid & \text { s.t. } x[x, u(\cdot), v(\cdot)](\tau) \in \mathcal{E} \text { if } \tau<+\infty, \text { and } \\
& \forall t \in[0, \tau), x[x, u(\cdot), v(\cdot)](t) \in K_{n}
\end{array}\right\}
$$

It is easy to check that $\bigcap_{n} K_{n}$ is a discriminating domain. Hence $\bigcap_{n} K_{n} \subset$ $\operatorname{Disc}_{H}(\mathcal{K})$. On another hand, since the $K_{n}$ contain $\operatorname{Disc}_{H}(\mathcal{K})$, one has $\bigcap_{n} K_{n}=$ $\operatorname{Disc}_{H}(\mathcal{K})$. Hence $\bigcap_{n} K_{n}=\operatorname{Disc}_{H}(\mathcal{K})$.

For the characterization of Ursula's victory domain, we introduce the complement of Ursula's victory domain:

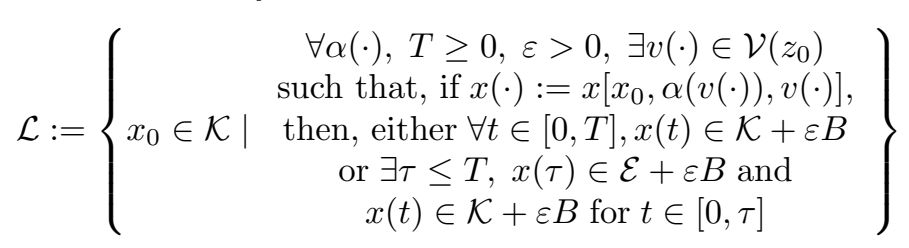

Thanks to Theorem 2.4, $\operatorname{Disc}_{H}(\mathcal{K}) \subset \mathcal{L}$. So we have to prove the converse inclusion.

For doing this, the key argument of [10] (Lemma 5.1) is replaced by the following

Lemma 4.3 If $x_{0}$ belongs to $\mathcal{K}$ but not to $\mathcal{L}$, there are positive $\eta$, $\varepsilon$ and $T$ and, for any $x:=(y, z) \in\left(x_{0}+\eta B\right) \cap \mathcal{K}$, a non-anticipative strategy $\alpha: \mathcal{V}(z) \rightarrow \mathcal{U}(y)$ such that, for any $v(\cdot) \in \mathcal{V}(z)$, the solution $x[x, \alpha(v(\cdot)), v(\cdot)]$ does not reach $\mathcal{E}+\varepsilon B$ and leaves $\mathcal{K}+\varepsilon B$ before $T$. Namely, if we set $x(\cdot):=x[x, \alpha(v(\cdot)), v(\cdot)]$,

$$
\exists \tau \in[0, T], x(\tau) \notin \mathcal{K}+\varepsilon B \text { and } x(t) \notin \mathcal{E}+\varepsilon B \text { for } t \in[0, \tau] .
$$

Lemma 4.3 states that the $\varepsilon$ and $T$ appearing in the Definition of Ursula's victory domain are locally uniform.

Proof of Lemma 4.3 : From the very definition of $\mathcal{L}$, if $x_{0}$ does not belong to $\mathcal{L}$, there is a non-anticipative strategy $\alpha_{0}(\cdot), T$ and $\varepsilon_{0}>0$ such that for any $v(\cdot) \in \mathcal{V}\left(z_{0}\right)$, if we set $x_{0}(\cdot):=x\left[x_{0}, \alpha_{0}(v(\cdot)), v(\cdot)\right]$,

$$
\exists \tau \in[0, T], x_{0}(\tau) \notin \mathcal{K}+\varepsilon_{0} B \text { and } x_{0}(t) \notin \mathcal{E}+\varepsilon_{0} B \text { for } t \in[0, \tau] .
$$

In the proof of Lemma 5.1 of [10], the same strategy $\alpha_{0}(\cdot)$ gave the desired result for any $x \in\left(x_{0}+\eta B\right)$ - provided that $\eta$ was sufficiently small. The situation is more complicated here because $\alpha_{0}$ is not necessarily an admissible strategy for $x \in\left(x_{0}+\eta B\right) \cap \mathcal{K}$ because of the constraints. For solving this difficulty, we use the following Lemma proved below. Although we shall apply this Lemma indifferently to $y$ and to $z$, we only formulate it for $y$. 
Lemma 4.4 Let $Q$ and $T$ be fixed positive constants. There is some $\lambda>0$ (depending on the constants of the problem on $Q$ and on $T$ ) such that, for any $y$ and $y_{0}$ belonging to $K_{U}$, with $\|y\| \leq Q$ and $\left\|y_{0}\right\| \leq Q$, there is a non-anticipative strategy $\sigma: \mathcal{U}(y) \rightarrow \mathcal{U}\left(y_{0}\right)$ with, for any $u(\cdot) \in \mathcal{U}(y)$, for $t \in[0, T]$,

$$
\left\|y\left[y_{0}, \sigma(u(\cdot))\right](t)-y[y, u(\cdot)](t)\right\| \leq\left\|y-y_{0}\right\| e^{\lambda t} .
$$

Let us complete the proof of Lemma 4.3. We fix a constant $Q$ sufficiently large in such a way that any solution starting from a point of $x_{0}+B$ remains in the ball $Q B$ on $[0, T]$. Fix $\eta:=\varepsilon_{0} e^{-\lambda T} / 4$, where $\lambda$ is defined by Lemma 4.4. Let $x:=(y, z) \in\left(x_{0}+\eta B\right)$.

From Lemma 4.4, there is a non-anticipative map $\sigma_{1}: \mathcal{V}(z) \rightarrow \mathcal{V}\left(z_{0}\right)$ such that, for any $v(\cdot) \in \mathcal{V}(z)$, for any $t \in[0, T]$,

$$
\left\|z[z, v(\cdot)](t)-z\left[z_{0}, \sigma_{1}(v(\cdot))\right](t)\right\| \leq \eta e^{\lambda t} .
$$

Then $\alpha_{0} \circ \sigma_{1}: \mathcal{V}(z) \rightarrow \mathcal{U}\left(y_{0}\right)$ is a non-anticipative map. From Lemma 4.4 again, there is a non-anticipative strategy $\sigma_{2}: \mathcal{U}\left(y_{0}\right) \rightarrow \mathcal{U}(y)$ such that, for $t \in[0, T]$,

$$
\left\|y\left[y_{0}, u(\cdot)\right](t)-y\left[y, \sigma_{2}(u(\cdot))\right](t)\right\| \leq \eta e^{\lambda t}
$$

for any $u(\cdot) \in \mathcal{U}(y)$. Then $\alpha:=\sigma_{2} \circ \alpha_{0} \circ \sigma_{1}$ is a non-anticipative strategy from $\mathcal{V}(z)$ to $\mathcal{U}(y)$.

Fix some control $v(\cdot) \in \mathcal{V}(z)$. Set $x_{0}(\cdot):=x\left[x_{0}, \alpha_{0}\left(\sigma_{1}(v(\cdot))\right), \sigma_{1}(v(\cdot))\right]$ and $x(\cdot):=x[x, \alpha(v(\cdot)), v(\cdot)]$.

Then, for $\tau \in[0, T]$ defined in formula (10),

$$
d_{\mathcal{K}}(x(\tau)) \geq d_{\mathcal{K}}\left(x_{0}(\tau)\right)-\left\|x(\tau)-x_{0}(\tau)\right\| \geq \varepsilon_{0}-2 \eta e^{\lambda T} \geq \varepsilon_{0} / 2 .
$$

In the same way, $d_{\mathcal{E}}(x(t)) \geq \varepsilon_{0} / 2$ on $[0, \tau]$, which completes the proof of Lemma 4.3 .

Proof of Lemma 4.4 : From Lemma 1.1, there is some $\lambda>0$ such that, for any $u_{0}(\cdot) \in \mathcal{U}\left(y_{0}\right)$, for any $y \in K_{U}$, there is some control $u(\cdot) \in \mathcal{U}(y)$ with

$$
\left\|y[y, u(\cdot)](t)-y\left[y_{0}, u_{0}(\cdot)\right](t)\right\| \leq\left\|y-y_{0}\right\| e^{\lambda t}
$$

for any $t \in[0, T]$. Let us consider the set-valued map $\Sigma: \mathcal{U}\left(y_{0}\right) \rightarrow \mathcal{U}(y)$ defined by

$$
\Sigma\left(u_{0}(\cdot)\right):=\{u(\cdot) \in \mathcal{U}(y) \mid(11) \text { is satisfied }\}
$$

It is easy to check that $\Sigma$ is non-anticipative in the sense of [14], so that, from Plaskacz Lemma (Lemma 2.7 of [14]), it enjoys a non-anticipative selection $\sigma$, i.e., $\sigma$ is non-anticipative and $\sigma\left(u_{0}(\cdot)\right) \in \Sigma\left(u_{0}(\cdot)\right)$ for any $u_{0} \in \mathcal{U}\left(y_{0}\right)$.

\section{References}

[1] ALZIARY de ROQUEFORT (1991) Jeux différentiels et approximation numérique de fonctions valeur, RAIRO Math. Model. Numer. Anal., 25, pp. 517-560.

[2] ARISAWA M. \& LIONS P.L. (1996) Continuity of admissible trajectories for state constraints control problems, Discrete Contin. Dynam. Systems, 2, no. 3, 297-305. 
[3] AUBin J.-P. (1991) Viability Theory. Birkhäuser.

[4] AUBin J.-P.\& FRANKOWSKA H. (1990) Set-valued analysis. Birkhäuser.

[5] BARDi M., BOTTACIN S. \& FALCONE M. (1995) Convergence of discrete schemes for discontinuous value functions of pursuit-evasion games. New trends in dynamic games and applications, 273-304, Ann. Internat. Soc. Dynam. Games, 3, Birkhpp. 273304.

[6] BARDI M., KOIKE S. \& SORAVIA P. (1998) Pursuit-evasion game with state constraints: dynamic programming and discrete-time approximations, Pre-print.

[7] BERKOVITZ L. (1986) Differential games of generalized pursuit and evasion, SIAM J. Control and Optimization, Vol. 24, N. 3

[8] BERNHARD P. (1979) Contribution à l'étude des Jeux Différentiels à deux joueurs, somme nulle, et information parfaite Thèse de Doctorat d'Etat, Université Pierre et Marie Curie - Paris 6.

[9] BREAKWELL J.V. (1977) Zero-sum differential games with terminal payoff. In Differential Game and Applications, Hagedorn p., Knobloch H.W. \& Olsder G.H. Ed Lecture Notes in Control and Information Sciences Vol.3, Springer Verlag.

[10] CARDAliagueT P. (1996) A differential game with two players and one target, SIAM J. Control and Optimization, Vol. 34, N. 4, pp. 1441-1460.

[11] CARDALIAGUET P. (1997) Non smooth semi-permeable barriers, Isaacs equation and application to a differential game with one target and two players, Appl. Math. Opti., 36, pp. 125-146.

[12] CARDALIAGUET P. QUINCAMPOIX M. \& SAINT-PIERRE P (1995) Contribution à l'étude des jeux différentiels quantitatifs et qualitatifs avec contraintes sur l'état, C.R. Acad .Sci. Paris, t. 321, Série I, pp. 1543-1548.

[13] CARDAliagueT P., QUINCAMPOIX M. \& SAINT-PIERRE P. (1999) Numerical methods for differential games, in "Stochastic and differential games: Theory and numerical methods", pp. 177-247. Annals of the international Society of Dynamic Games, M. Bardi, T.E.S. Raghavan, T. Parthasarathy Eds. Birkhäuser.

[14] CARDALIAGUET P. \& PLASKACZ S. (to appear) Invariant solutions of differential games and Hamilton-Jacobi equations for time-measurable hamiltonians, SIAM Journal on Control and Optim.

[15] ELLIOT N.J. \& KALTON N.J. (1972) The existence of value in differential games Mem. Amer. Math. Soc., 126.

[16] ELLIOT N.J. \& KALTON N.J. (1972) The existence of Value in Differential Games of Pursuit and Evasion J. Differential Equations, Vol 12, pp. 504-523.

[17] EVANS L.C. \& SOUGANDINIS P.E. (1984) Differential games and representation formulas for solutions of Hamilton-Jacobi Equations Indiana Univ. Math. J., 282, pp. 487-502.

[18] FLEMING W. H. (1967) The convergence problem for differential games, J. Math. Anal. and Appl., Vol. 3, pp. 102-116

[19] FLYNN J. (1973) Lion and Man : The Boundary Constraints, SIAM J. on Control, 11, p.397

[20] FRANKOWSKA H., PLASCASZ M. \& RZEZUCHOWSKI T. (1995) Measurable Viability Theorem and Hamilton-Jacobi-Bellman Equations, J. Diff. Eqs., Vol. 116, No 2, pp. 265-305.

[21] FRANKOWSKA H \& RAMPAZZO F. (2000) Filippov's and Filippov-Wazewski's theorems on closed domains, J. Differential Equations 161, no. 2, 449-478.

[22] FRIEDMAN A. (1970) On the definition of differential games and the existence of value and saddle points, J. Differential equations 7, pp. 69-91 
[23] FRIEDMAN A. (1970) Existence of value and saddle points for differential games of pursuit and evasion, J. Differential equations 7, pp. 92-125

[24] FRIEDMAN A. (1970) Differential games with restricted phase coordonates, J. Differential equations, 8, pp. 135-162.

[25] ISAACS R. (1965) Differential Games Wiley, New York.

[26] Krasovskit N.N. \& SUbBotin A.I. (1988) Game-Theorical Control Problems Springer-Verlag, New-York.

[27] LORETI P. \& TESSITORE M.E. (1994) Approximation and regularity results on constrained viscosity solutions of Hamilton-Jacobi-Bellman equations. J. Math. Systems Estim. Control 4, no. 4, pp. 467-483.

[28] OSIPOV Ju. S. (1971) Alternative in a Differential-Difference Game, Soviet Math. Dokl., Vol. 12, pp. 619-624.

[29] PLASKACZ S. (1994) Personnal communication

[30] PETROV N.N. (1970) On the existence of a value for pursuit games, Soviet Mat. Dokl., 11, pp. 292-294

[31] ROXIN E. (1969) The axiomatic approach in differential games, J. Optim. Theory Appl. 3, pp. 153-163.

[32] ROZYEV I. \& SUBBOTIN A.I. (1988) Semicontinuous solutions of Hamilton-Jacobi Equations. PMM U.S.S.R., Vol. 52, N. 2, pp. 141-146.

[33] SONER M.H. (1986) Optimal control problems with state-space constraints. SIAM J. on Control and Optimization, 24, pp. 552-562 and pp.1110-1122.

[34] SORAVIA P. (1993) Pursuit-evasion games and viscosity solutions of Isaacs equation, SIAM J. Control Optim., 31, pp. 604-623.

[35] VARAIYA P. (1967) The existence of solution to a differential game, SIAM J. Control Optim. 5, pp. 153-162.

[36] VARAIYA P. \& LIN J. (1967) Existence of Saddle Points in differential game, SIAM J. Control Optim. 7, No 1, pp. 141-157.

[37] YONG J. (1988) On differential pursuit games, SIAM J. Control Optim., 26, pp. 478495 . 


\section{Contents}

1 Existence of a value for the pursuit game $\quad 3$

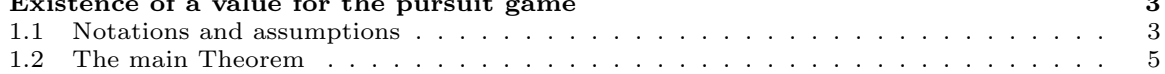

2 An Alternative Theorem for a qualitative differential game with state constraints 7

2.1 Statement of the qualitative problem . . . . . . . . . . . . . . . . . 8

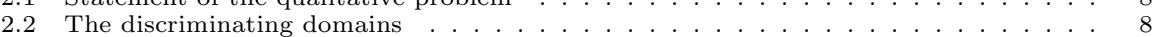

2.3 The Alternative Theorem . . . . . . . . . . . . . . . . . . . . . . . . . . . . . . . . . . . . . . . . . . . . . . . . . . . . . . .

3 Proof of the existence of a value for the pursuit game 10

4 Appendix 13

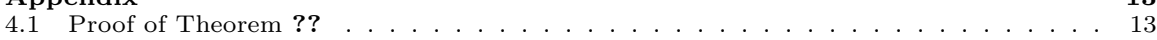

15

4.3 Proof of Theorem ?? . . . . . . . . . . . . . . . . . 18 